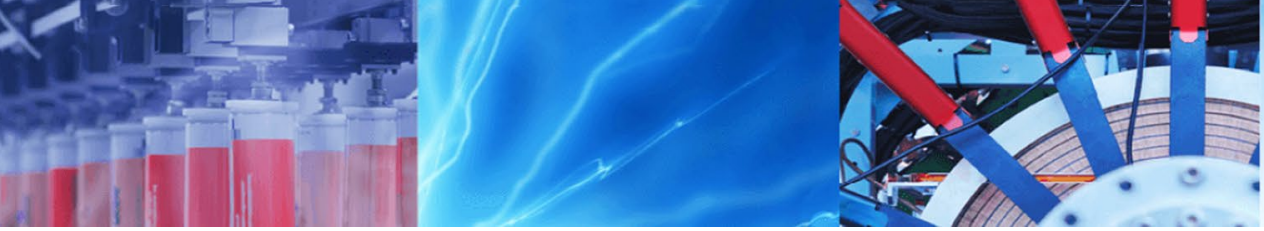

Research Article

\title{
Time-based damage detection of underground ferromagnetic pipelines using complexity pursuit based blind signal separation
}

\author{
Zia Ullah $^{1,2}$ - Xinhua Wang ${ }^{1} \cdot$ Yingchun Chen $^{1} \cdot$ Tao Zhang $^{1} \cdot$ Haiyang Ju ${ }^{1} \cdot$ Yizhen Zhao $^{1}$
}

(c) Springer Nature Switzerland AG 2019

\begin{abstract}
The measured sensor data of underground ferromagnetic pipelines consist of hidden damage information that can be explored by developing output data identification models due to the availability of only output signal responses. The current research findings elaborate output-only modal identification method Complexity Pursuit based on blind signal separation. An attempt is made to apply the complexity pursuit algorithms, for time-based damage detection of underground ferromagnetic pipelines which blindly estimates the modal parameters from the measured magnetic field signals for targeting the pipeline defect locations. Numerical simulations for multi-degree of freedom systems show that the proposed tested method could precisely identify the structural parameters. Experiments are conducted primarily under well-equipped controlled laboratory conditions followed by confirmation in the real field on pipeline magnetic field data, recorded through high precision magnetic field sensors. The measured recorded structural responses are given as input to the blind source separation model where the complexity pursuit algorithms blindly extracted the least complex signals from the observed mixtures guaranteed to be source signals. The output power spectral densities calculated from the estimated modal responses unveiled elegant physical interpretation of the underground ferromagnetic pipeline structures.
\end{abstract}

Keywords Blind source separation · Pipeline flaw detection · Structure health monitoring · Complexity pursuit · Output modal identification

$\begin{array}{ll}\text { Abbreviations } \\ \text { BSS } & \text { Blind Source Separation } \\ \text { CP } & \text { Complexity Pursuit } \\ \text { CP-BSS } & \text { Complexity Pursuit-Blind Source Separation } \\ \text { CPU } & \text { Central Processing Unit } \\ \text { DC } & \text { Direct Current } \\ \text { DOF } & \text { Degree of Freedom } \\ \text { GPS } & \text { Global Positioning System } \\ \text { GWN } & \text { Gaussian White Noise } \\ \text { MAC } & \text { Modal Assurance Criterion } \\ \text { NDT } & \text { Non-Destructive Testing } \\ \text { PSD } & \text { Power Spectral Density } \\ \text { SDOF } & \text { Single Degree of freedom }\end{array}$

\section{Introduction}

Pipelines are important channels of oil and gas transportation. They are buried underground for the reason of safety, ease of operation and most important to prevent the influence of traffic and farming activities [1]. They are made of ferromagnetic materials which are often vulnerable to corrosion, fatigue damages and cracks due to surrounding environmental effects such as transmission channels, soil, temperature variations and other mechanical flaws; hence, require early damage detection techniques to confirm safe operation and reliable energy supply [2]. To achieve such goals, the primary aim of this study is to develop a

$\triangle$ Zia Ullah, ziaullah@emails.bjut.edu.cn; Xinhua Wang, wangxinhua@bjut.edu.cn; Yingchun Chen, ychen08089@163.com; Tao Zhang, zhangtao1989@emails.bjut.edu.cn; Haiyang Ju, juhaiyang@emails.bjut.edu.cn; Yizhen Zhao, zhyizh@emails.bjut.edu.cn | ${ }^{1}$ College of Mechanical Engineering, Beijing University of Technology, Beijing, People's Republic of China. ${ }^{2}$ College of Mechanical Engineering and Applied Electronics Technology, Beijing University of Technology, No 100 Ping Le Yuan Chaoyang District, Beijing 100124, People's Republic of China. 
non-contact geomagnetic probe using basic principles of metal magnetic memory testing $[3,4]$ to detect the geomagnetic field signals. Non-contact pipeline magnetic field testing [5] is a new kind of non-destructive testing (NDT) technique that needs earth magnetic field as the stimulus source to locate underground ferromagnetic pipelines and achieves structural defect information i.e. crack, corrosion and dent etc. without any excavation. However, the magnetic field data recorded for large scale systems like underground pipelines are often contaminated by several factors when in their service environment, as: the interference of parallel communication lines along the pipeline in heavy traffic areas, unusual disturbance often caused by the underground subway passages and overhead high voltage lines. Considerable attention in developing such non parametric methods than can perform quick real-time assessment of the 3-axis magnetic field data is required towards safety and integrity of pipelines.

Several signal processing techniques [6-14] have been considered in the literature for modal identification and flaw detection. Recently, blind source separation (BSS) techniques have been used as promising signal analysis tools in various fields of science [15-19]. BSS based algorithms are computational methods used for separating a multivariate signal into additive subcomponents. These methods were successfully applied for damage identification of civil structures [20-22] and more recently for flaw detection of underground ferromagnetic pipelines [23].

This paper presents a time-domain output data identification model for pipeline magnetic field data using the unsupervised blind source separation technique termed complexity pursuit (CP) [24] that was independently formulated in [25]. CP learning algorithms have been successfully applied for system identification and damage detection in [26-30]. The main contribution of this paper is to apply the $\mathrm{CP}$ algorithms to the pipelines noisy magnetic field data, towards an accurate time-based modal identification. The 3-axis magnetic field data are fed as input into the blind source separation model where the complexity pursuit algorithms are applied for an accurate extraction of mode matrix that is then plotted to obtain the time-domain output modal responses. The power spectral densities calculated from the recovered mode matrix show the abrupt variation in frequency due to the defects occurring in the pipeline. The detailed indoor and outdoor experimental results show the ability of the non-parametric CP-BSS learning algorithms to accurately extract time based modal information of the pipeline structures.

\section{Blind source separation problem}

The process of identifying and extracting the original source signals from a mixture of signals with less amount of information about the original source signals are termed as blind source separation.

The linear instantaneous blind source separation model is written by Ref. [25] as:

$\mathbf{x}(t)=\mathbf{A} \mathbf{s}(t)=\sum_{i=1}^{n} a_{i} s_{i}(t)$

where $\mathbf{x}(t)=\left[x_{1}(t), \ldots, x_{m}(t)\right]^{T}$ is the measured signal, containing $m$ mixture signals, and $\mathbf{s}(t)=\left[s_{1}(t), \ldots, s_{n}(t)\right]^{T}$ is the original source vector with $n$ sources; $\mathbf{A} \in \mathrm{R}^{m \times n}$ is an unknown matrix consisting of $n$ columns with its ith column $a_{i} \in \mathrm{R}^{m}$ associated with $s_{i}(t)$.

\section{Stone's theorem for solution of blind source separation problem}

Stone [24] proposed that under the effect of some physical laws; the moment of mass in a given time produces possible sources in a system. Likewise, the measured system responses also contains least complex sources, each source is created under the influence of certain physical law. Summarizing, the complexity of a mixture of response signals can be found among the simplest and the most composite constituent sources. It was theoretically proved in [31].

This conclusion laid the foundation that source signals are the least complex signals that can be separated from the measured mixture of signals. Thus complexity pursuit algorithms search for source signals with least complexity, such that the "hidden" source component $z_{i}(t)$ is obtained by multiplying the mixture $\mathbf{x}(t)$ with the demixing row vector $w_{i}$ which is the least complex signal.

$z_{i}(t)=w_{i} \mathbf{x}(t)$

This approach has been used as a solution towards the BSS problem.

Stone [24] concluded that the complexity of a signal can be measured by maximizing the temporal predictability of a signal; the mathematical equation for temporal predictability is written as:

$F\left(z_{i}\right)=\log \frac{V\left(z_{i}\right)}{U\left(z_{i}\right)}=\log \frac{\sum_{t=1}^{N}\left(z_{L}(t)-z_{i}(t)\right)^{2}}{\sum_{t=1}^{N}\left(z_{S}(t)-z_{i}(t)\right)^{2}}$ 
$\mathrm{F}\left(z_{i}\right)$ is the temporal predictability operator that contains the statistical and time-based information of the hidden source signal $z_{i}(t)$, that can be measured by finding the logarithmic ratio of $V\left(z_{i}\right) / U\left(z_{i}\right)$.

The term $V\left(z_{i}\right)$ determines the 'global statistical information' of signal $z_{i}(t)$ by computing the 'overall variability' estimated by a long-range prediction parameter $z_{L}(t)$. Similarly, $U\left(z_{i}\right)$ determines the 'local variance' that calculates the time-based information [26], by using a short-range prediction parameter $z_{S}(t)$ on the temporal structure of signal $z_{i}(t)$. The filtration process is performed by the long range prediction parameter and short range prediction parameter, mathematically expressed as,

$\mathrm{z}_{L}(t)=\lambda_{L} z_{L}(t-1)+\left(\left(1-\lambda_{L}\right) z_{i}(t-1)\right) 0 \leq \lambda_{L} \leq 1$

$z_{S}(t)=\lambda_{S} z_{S}(t-1)+\left(\left(1-\lambda_{S}\right) z_{i}(t-1)\right) 0 \leq \lambda_{S} \leq 1$

$\lambda=2^{-1 / h}$ where $h$ is termed as a half-life parameter for $h_{S}=1$ and $h_{L}=900,000$ as long as $h_{L} \gg h_{\mathrm{S}}$ [25].

The significance of the proposed algorithm is to extract the hidden sources with accurate time-based structure. Since the use of short and long range prediction parameters should be considered carefully; as 'increasing only the global statistical information $V\left(z_{i}\right)$ will produce a high variance signal.' While 'increasing only $U\left(z_{i}\right)$ will produce a smooth DC signal.'Thus careful selection of parameters is essential to predict a component with reduced local variance (smoothness) as compared with its global (longrange) variance.

\subsection{System identification by complexity pursuit}

Combining Eqs. (2) and (3)

$\mathrm{F}\left(z_{i}\right)=\mathrm{F}\left(\mathrm{w}_{i}, \mathbf{x}\right)=\log \frac{V\left(w_{i}, \mathbf{x}\right)}{U\left(w_{i}, \mathbf{x}\right)}=\log \frac{w_{i} \overline{\mathbf{P}} w_{i}^{T}}{w_{i} \hat{\mathbf{P}} w_{i}^{T}}$

where $\overline{\mathbf{P}}$ and $\hat{\mathbf{P}}$ are the $M \times M$ short-range and long-range covariance matrices among the mixtures, respectively. The elements of these matrices are given by;

$\hat{p}_{i j}=\sum_{t=1}^{N}\left(p_{i}(t)-\hat{p}_{i}(t)\right)\left(p_{j}(t)-\hat{p}_{j}(t)\right)$

$\bar{p}_{i j}=\sum_{t=1}^{N}\left(p_{i}(t)-\bar{p}_{i}(t)\right)\left(p_{j}(t)-\bar{p}_{j}(t)\right)$

The matrices $\overline{\mathbf{P}}$ and $\hat{\mathbf{P}}$ are calculated only once and the terms $\left(p_{i}(t)-\hat{p}_{i}(t)\right)$ and $\left(p_{j}(t)-\bar{p}_{j}(t)\right)$ are calculated by fast convolution operations. For a given mixture of signals $\mathbf{x}(t)$, the complexity pursuit algorithm calculates the de-mixing vector $w_{i}$ by maximizing the temporal predictability function $\mathrm{F}\left(z_{i}\right)$;

The derivative of $\mathrm{F}$ with respect to $w_{i}$ is given by

$\nabla_{w_{i}} \mathrm{~F}=\frac{2 w_{i}}{V_{i}} \overline{\mathbf{P}}-\frac{2 w_{i}}{U_{i}} \hat{\mathbf{P}}$

Using the gradient ascent technique a maximum value of $\mathrm{F}$ can be obtained by repeatedly updating $w_{i}$; such that the extracted component $z_{i}=w_{i} \mathbf{x}$, which is "most predictable" is considered as the least complex signal or the simplest source hidden in the mixtures [24].

Considering the uncertainties of the proposed CP model for extracting only one simplest source can be solved by the deflation scheme [24]. The sources are simultaneously extracted one after another using Gram-Schmidt de-correlation technique. The first step is to separate the most simplest source present in the mixture, after removing the first source the currently simplest source becomes the second one to be separated by the complexity pursuit algorithm and so on. The solution for gradient of $F$ approaches zero such that,

$\nabla_{w_{i}} \mathrm{~F}=\frac{2 w_{i}}{V_{i}} \overline{\mathbf{P}}-\frac{2 w_{i}}{U_{i}} \hat{\mathbf{P}}=0$.

Rearranging Eq. (8)

$w_{i} \overline{\mathbf{P}}=\frac{V_{i}}{U_{i}} w_{i} \hat{\mathbf{P}}$

Equation (9) has the form of a generalized eigenproblem; $w_{i}$ can be found as the eigenvectors of matrix $\hat{\mathbf{P}}^{-1} \overline{\mathbf{P}}$, with corresponding eigenvalues $\gamma_{i}=V_{i} / U_{i}$. The de-mixing matrix $\mathbf{A}=\mathbf{W}^{-1}$ is calculated using a generalized eigenvalue routine. All the source signals can be separated by,

$\mathbf{s}(t)=\mathbf{z}(t)=\mathbf{W} \mathbf{x}(t)$

$\mathbf{s}(t)=\left[s_{1}(t), \ldots, s_{n}(t)\right]^{T}$ is the recovered source matrix with row-wise source signals $s_{i}(t)$.

\subsection{Modal parameters estimated by complexity pursuit}

The connection of blind source separation with output modal identification was solved for the first time in [22], which stated that the governing equation of motion for a linear time invariant system is given by,

$\mathbf{M} \ddot{\mathbf{x}}+\mathbf{C} \dot{\mathbf{x}}(t)+\mathbf{K x}(t)=\mathbf{f}(t)$

where $\mathbf{M}$ is mass, $\mathbf{C}$ is the damping matrix and $\mathbf{K}$ is the stiffness matrix, all real valued and symmetric. $x(t)$ is the displacement vector, which are actually the measured system responses. $f(t)$ is the external force acting on the system. 
Same like the BSS model in Eq. (1) the modes of a system can be expanded as linear combination of $n$ number of modal responses that can be expressed in Eq. (12) as;

$\mathbf{x}(t)=\Phi \mathbf{q}(t)=\sum_{i=1}^{\mathrm{n}} \varphi_{i} q_{i}(t)$

The basic phenomenon of Eq. (12) is same as in Eq. (1), $\boldsymbol{\Phi}$ contains the modal information of a system describing the entire situation of a noise contaminated system. $\varphi_{i} \in \mathbb{R}^{n}$ being the (mode shape) is the related to ith modal feature column of the mode matrix, and is related with the $i$ th modal response $q_{i}(t)$ of the modal response vector $\mathbf{q}(t)$. $\mathbf{q}(t)$ is actually the original source signal that can be obtained by multiplying the inverse of the mode matrix with the $(m \times n)$ matrix of the measured system responses.

$\mathbf{q}(t)=\boldsymbol{\Phi}^{-1} \mathbf{x}(t)$

$\Phi \in \mathbb{R}^{n x n}$ is used to identify the change in the normal condition of a system as an abrupt variation in the modal feature. Thus, plotting the mode shapes can give important information about the damage occurring in the system under observation.

The idea of "virtual sources" in [22] states that the recovered modal responses of a system should be considered as independent sources, if the power spectral density is not same or the frequencies are not able to be judged clearly. In such cases the mixing matrix matches with the recovered modal matrix, consequently the hidden sources and unidentified mixing matrix can be obtained by putting the measured system responses from the expanded model in Eq. (12) as known mixtures into the blind source separation framework in Eq. (1); accordingly the desired modal responses and mode matrix can be achieved.

Equation (12) can be used to classify the motion of a system by its mode matrix $\boldsymbol{\Phi}$ as it provides complete information about the linear system. Putting Eq. (12) into Eq. (11) and multiplying the transpose of the mode matrix $\boldsymbol{\Phi}^{T}$ on both sides,

$\boldsymbol{\Phi}^{T} \mathbf{M} \Phi \ddot{\mathbf{q}}(t)+\boldsymbol{\Phi}^{T} \mathbf{C} \Phi \dot{\mathbf{q}}(t)+\boldsymbol{\Phi}^{T} \mathbf{K} \Phi \mathbf{q}(t)=\boldsymbol{\Phi}^{T} \mathbf{f}(t)$

yield to:

$\mathbf{M}^{*} \ddot{\mathbf{q}}(t)+\mathbf{C}^{*} \dot{\mathbf{q}}(t)+\mathbf{K}^{*} \mathbf{q}(t)=\mathrm{f}^{*}(t)$

where $\mathbf{M}^{*}$ is the diagonal real-valued modal mass matrix, $\mathbf{C}^{*}$ is damping matrix, and $\mathbf{K}^{*}$ is the stiffness matrix. $\mathbf{f}^{*}(t)$ is the modal force vector. A multi-DOF system can be decoupled into $n$-DOF systems whose motions are given by,

$m_{i}^{*} \ddot{q}_{i}(t)+c_{i}^{*} \dot{q}_{i}(t)+k_{i}^{*} q_{i}(t)=f_{i}^{*}(t)$

Equation (16) defines the basic idea of the complexity pursuit algorithm by targeting the motion of the decoupled single degree of freedom system on ith modal coordinate $q_{i}(t)$. The modal parameters of the system i.e. damping ratio is calculated by $\varsigma_{i}=c_{i}^{*} / 2 \sqrt{m_{i}^{*} k_{i}^{*}}$ and resonant frequency of the system is calculated in terms of natural frequency $\omega_{i}$ of the ith mode given by $\omega_{d i}=\omega_{i} \sqrt{1-\varsigma_{i}^{2}}=\sqrt{\left(1-\varsigma_{i}^{2}\right) k_{i}^{*} / m_{i}^{*}}$.

In case of no excitation i.e. $\mathrm{f}(t)=0$, the modal responses shall behave like a sinusoidal wave with an exponential decay. The motion of mass at ith modal coordinate governed by Eq. (16) can now be written as,

$q_{i}(t)=u_{i} e^{-\varsigma_{i} \omega_{i} t} \cos \left(\omega_{d i} t+\theta_{i}\right)$

These motions combine to form a linear combination of the measured responses given by,

$\mathbf{x}(t)=\Phi \mathbf{q}(t)=\sum_{i=1}^{n} \varphi_{i} q_{i}(t)=\sum_{i=1}^{n} \varphi_{i} u_{i} e^{-\varsigma_{i} \omega_{i} t} \cos \left(\omega_{d i} t+\theta_{i}\right)$

where $u_{i}$ and $\theta_{i}$ are some constants determined by initial conditions.

Kerschen et al. [22] found that in case of random excitation, the recovered modal responses are dominant over the measured system responses, that producing randomly modulated exponentially decaying sinusoids with an envelope function $e_{i}(t)$ at the $i$ th mode,

$q_{i}(t) \cong e_{i}(t) u_{i} e^{-\zeta_{i} \omega_{i} t} \cos \left(\omega_{d i} t+\theta_{i}\right)$

the measured responses are given by,

$\mathbf{x}(t)=\sum_{i=1}^{n} \varphi_{i} q_{i}(t) \cong \sum_{i=1}^{n} \varphi_{i} e_{i}(t) u_{i} e^{-\varsigma_{i} \omega_{i} t} \cos \left(\omega_{d i} t+\theta_{i}\right)$

In case of highly damped systems with complex valued mode matrix the complexity pursuit algorithms can separate the system into their respective modes until the intrinsic frequency and damping property of the system does not change. In such case the physical system in Eq. (12) can be decoupled into Eq. (17) in the state-space by the excitation mode matrix $\boldsymbol{\Phi}_{c}$, as well as the modal responses $\mathbf{q}_{c}(t)$. Therefore, using Stone's algorithm the measured mixture $\mathbf{x}(t)$ consisting of time based modal responses $\mathbf{q}(t)$, can be subsequently separated by CP-BSS model,

$\tilde{\mathbf{q}}(t)=\mathbf{s}(t)=\mathbf{W} \mathbf{x}(t)$

and the excitation mode matrix can be estimated by,

$\tilde{\Phi}=\mathbf{W}^{-1}$

The frequency and damping ratio can be readily computed from the recovered time-domain modal response $\tilde{\mathbf{q}}(t)$ using Fourier transform and logarithm-decrement technique, respectively. 


\section{Numerical simulations}

Using the $\mathrm{CP}$ based $\mathrm{BSS}$ algorithm, numerical examples are conducted on a 3-DOF system shown in Fig. 1 including different levels of damping.

The system parameters are adjusted to identify different modal identification problems i.e. proportional damping well separated mode, closely spaced mode and complex mode. Free excitation and random excitation in each case are discussed. Gaussian White Noise (GWN) is used to produce stationary random excitation [25]. Similarly the Gaussian White Noise (GWN) is modulated with a constant exponential decay function to create a non-stationary excitation effect in the system. The time histories of the system responses i.e. the displacement vector, are calculated by the Newmark-Beta solver. The sampling frequency is set to $10 \mathrm{~Hz}$.

The parameters of complexity pursuit based blind source separation method remains the same throughout the process. The long-range parameter $h_{L}=900,000$ and short-range parameter $h_{S}=1$ are taken same as given by [24]. Fast convolution operations are performed to calculate the long-range and short-range covariance matrices. The demixing matrix which is the eigenvector matrix is calculated by conducting eigenvalue decomposition on the obtained covariance matrices. The excitation mode matrix and the time-domain modal responses are calculated by Eqs. (21) and (22) respectively. Fourier transform algorithms and logarithm-decrement technique are used to calculate frequency and damping ratio respectively.

A modal assurance criterion is defined in Eq. (23) to evaluate the correlation among the recovered mode values $\tilde{\varphi}_{i}$ and the theoretical mode values $\varphi_{i}$.

$\operatorname{MAC}\left(\tilde{\varphi}_{i}, \varphi_{i}\right)=\frac{\left(\tilde{\varphi}_{i}^{T}, \varphi_{i}\right)^{2}}{\left(\tilde{\varphi}_{i}^{T} \cdot \tilde{\varphi}_{i}\right)\left(\varphi_{i}^{T} \cdot \varphi_{i}\right)}$

Ranging from 0 to 1 , where 0 means no correlation and 1 indicates perfect correlation.

\subsection{Proportional damping}

The parameters of the system shown in Fig. 2 are borrowed from Ref. [22]. In case of proportional damping

$$
\begin{aligned}
& \mathbf{M}=\left[\begin{array}{lll}
2 & 0 & 0 \\
0 & 1 & 0 \\
0 & 0 & 3
\end{array}\right] \quad \mathbf{K}=\left[\begin{array}{ccc}
2 & -1 & 0 \\
-1 & 2 & -1 \\
0 & -1 & 2
\end{array}\right] \\
& \mathbf{C}=\alpha \mathbf{M}=\alpha\left[\begin{array}{lll}
2 & 0 & 0 \\
0 & 1 & 0 \\
0 & 0 & 3
\end{array}\right]
\end{aligned}
$$

$\mathbf{M}$ is the mass matrix, $\mathbf{K}$ is the stiffness matrix and $\mathbf{C}$ is the damping matrix. The value of $\alpha$ corresponds to different damping level, ( $\alpha=0.03$ and 0.08). $\mathrm{f}(t)=0$ in free excitation with initial condition $\mathrm{x}(0)=\left[\begin{array}{lll}0 & 1 & 0\end{array}\right]^{T}$ and $\dot{x}(0)=\left[\begin{array}{lll}0 & 0 & 1\end{array}\right]^{T}$. In case of random excitation the system is excited at the $2^{\text {nd }}$ and $3^{\text {rd }}$ DOFs using stationary and nonstationary Gaussian White Noise. Tables 1 and 2 show the obtained results by $\mathrm{CP}$ algorithms and the modal assurance criterion values respectively.

Figure 2 shows the measured responses of a 3DOF linear system for $\alpha=0.08$ in free excitation proportional damping. After applying CP-BSS the estimated modes in free excitation are given in Figs. 3 and 4, respectively. The order of the recovered modes needs to be arranged as it shows the original modal responses recovered by the $\mathrm{CP}$ model for example the mode 1 in Fig. 3 means the first mode recovered by $\mathrm{CP}$ algorithm, not suggesting mode \#1), This is due to modal order problem that can be solved by rearranging the frequency values. Thus it can be observed that the measured responses of the 3DOF system are well-separated into their respective modes. The frequency of the separated modes can observed from the power spectral densities of the estimated modes.

\subsection{Effect of noise}

The calculated signal responses are now contaminated by adding zero-mean Gaussian White Noise (with a $10 \%$ of the original signal). The results for $\alpha=0.08$ in free excitation are shown in Table 3; addition of noise has no influence on the output of the CP model. Same accuracy has been seen in cases with various damping levels. It means that the $\mathrm{CP}$ algorithm provides healthy outputs for noise added signals also.
Fig. 1 The three-DOF linear spring-mass damped system

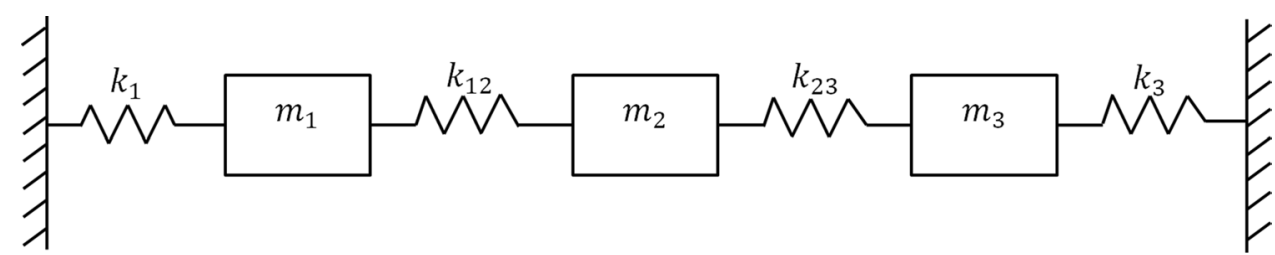

SN Applied Sciences a SPRINGER NATURE journa 
Fig. 2 Measured responses for 3DOF system in free excitation (proportional damping)
Table 1 Identified modal parameters (proportional damping)

Table 2 Identified MAC values (proportional damping)
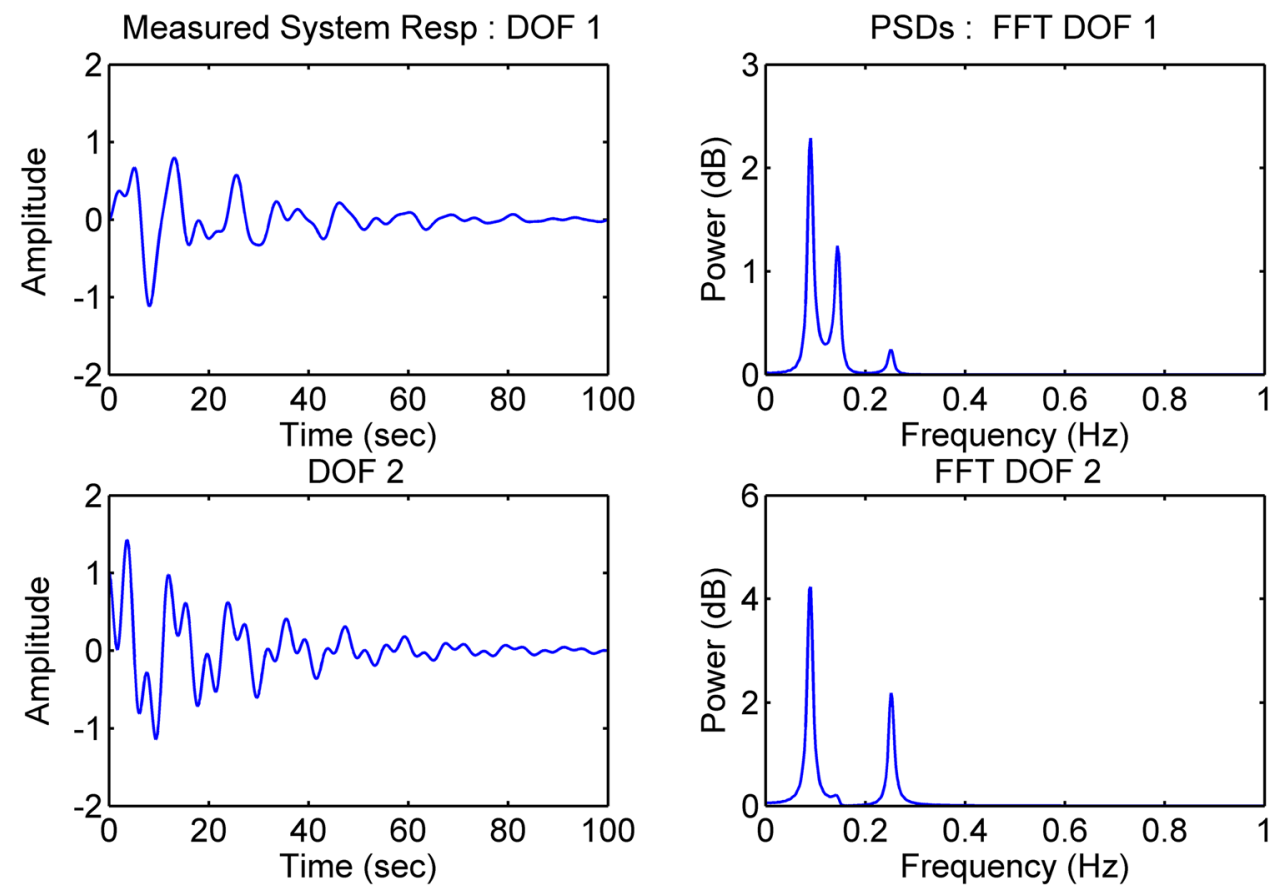

FFT DOF 2

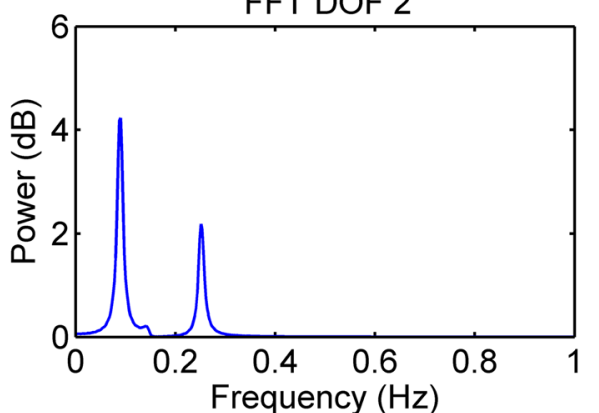

DOF 3
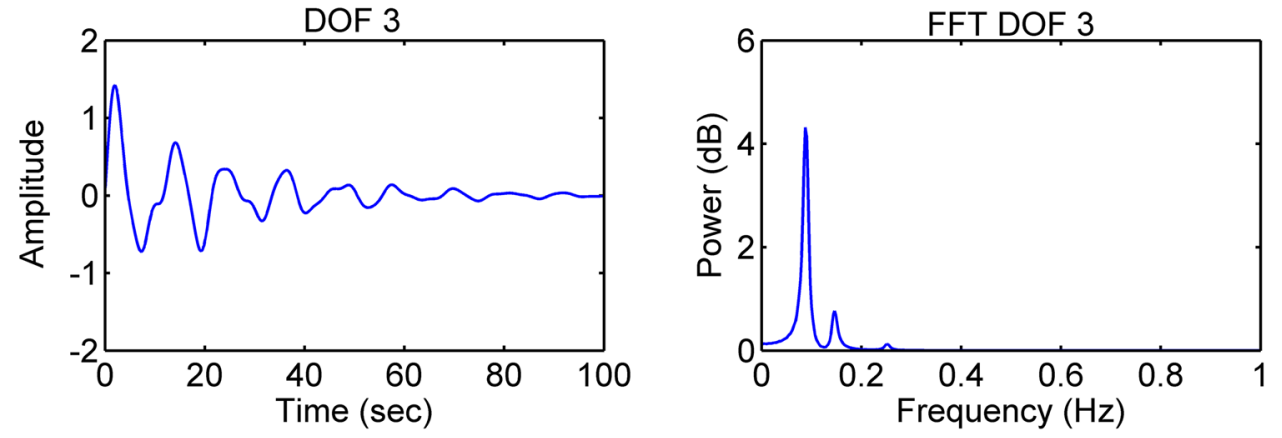

\begin{tabular}{|c|c|c|c|c|c|c|c|}
\hline \multirow[t]{2}{*}{ Mode } & \multirow[t]{2}{*}{ Comparison } & \multicolumn{3}{|c|}{ Frequency (Hz) } & \multicolumn{3}{|c|}{ Damping ratio (\%) } \\
\hline & & 1 & 2 & 3 & 1 & 2 & 3 \\
\hline \multirow[t]{2}{*}{$a=0.03$} & Theoretical value & 0.0895 & 0.1458 & 0.2522 & 0.8887 & 0.5460 & 0.3155 \\
\hline & CP identified value & 0.0879 & 0.1465 & 0.2539 & 0.8822 & 0.5691 & 0.3166 \\
\hline \multirow[t]{2}{*}{$a=0.08$} & Theoretical value & 0.0895 & 0.1458 & 0.2522 & 4.4437 & 2.7299 & 1.5775 \\
\hline & CP identified value & 0.0879 & 0.1465 & 0.2539 & 4.4493 & 2.8233 & 1.5248 \\
\hline
\end{tabular}

\begin{tabular}{|c|c|c|c|c|c|c|c|c|c|}
\hline \multirow[t]{2}{*}{ Mode } & \multicolumn{3}{|c|}{ Free excitation } & \multicolumn{3}{|c|}{ Stationary GWN } & \multicolumn{3}{|c|}{ Non-Stationary GWN } \\
\hline & 1 & 2 & 3 & 1 & 2 & 3 & 1 & 2 & 3 \\
\hline$a=0.03$ & 0.9988 & 0.9997 & 0.9992 & 1.0000 & 0.9997 & 0.9998 & 0.9997 & 0.9987 & 1.0000 \\
\hline$a=0.08$ & 0.9975 & 0.9993 & 0.9990 & 1.0000 & 0.9962 & 0.9997 & 0.9998 & 0.9977 & 0.9981 \\
\hline
\end{tabular}

\subsection{Closely spaced modes}

The complexity pursuit model was implemented on measured system responses of a 3DOF system with closely spaced modes. The mass, stiffness and damping matrix were obtained by modifying the high proportional damping matrix in [21]. 
Fig. 3 Recovered modes for 3DOF system in free excitation (proportional damping)
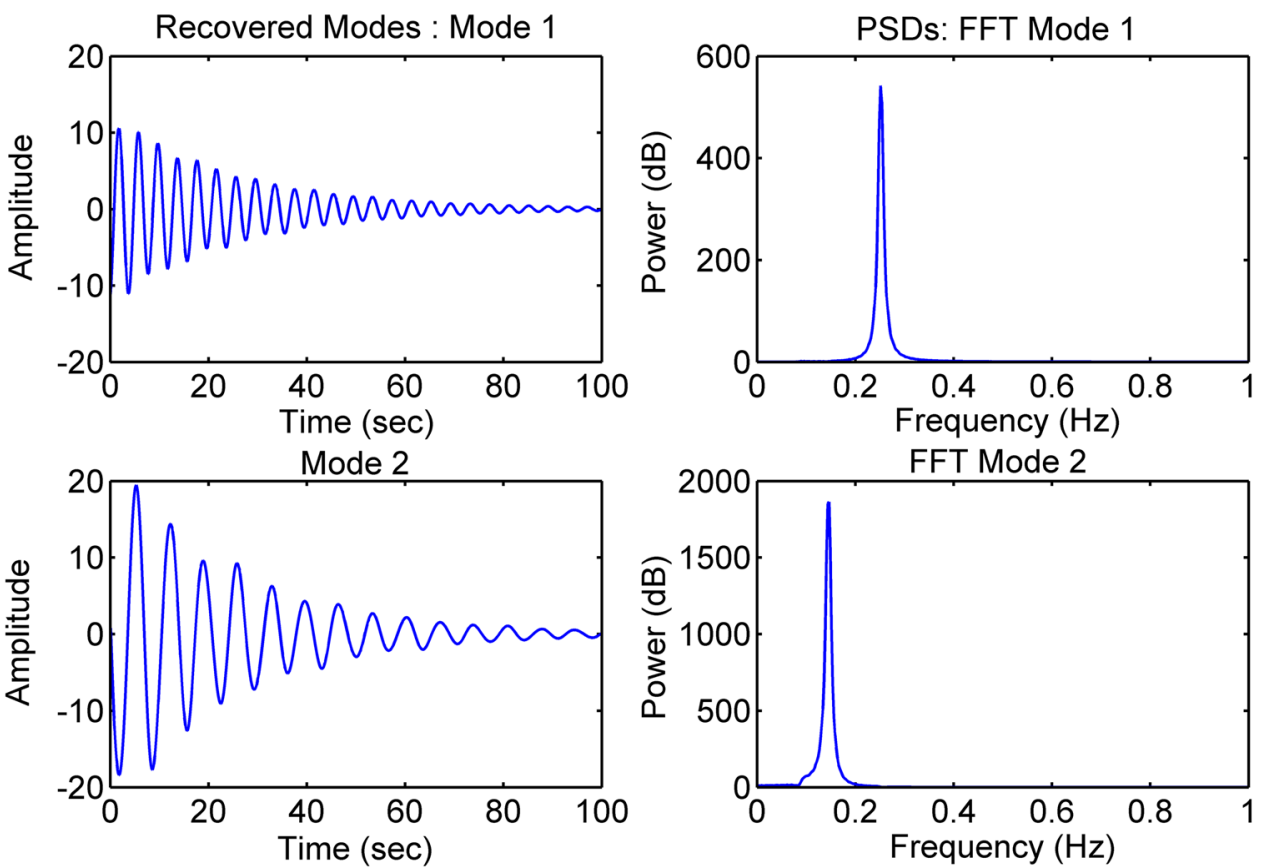

Mode 3
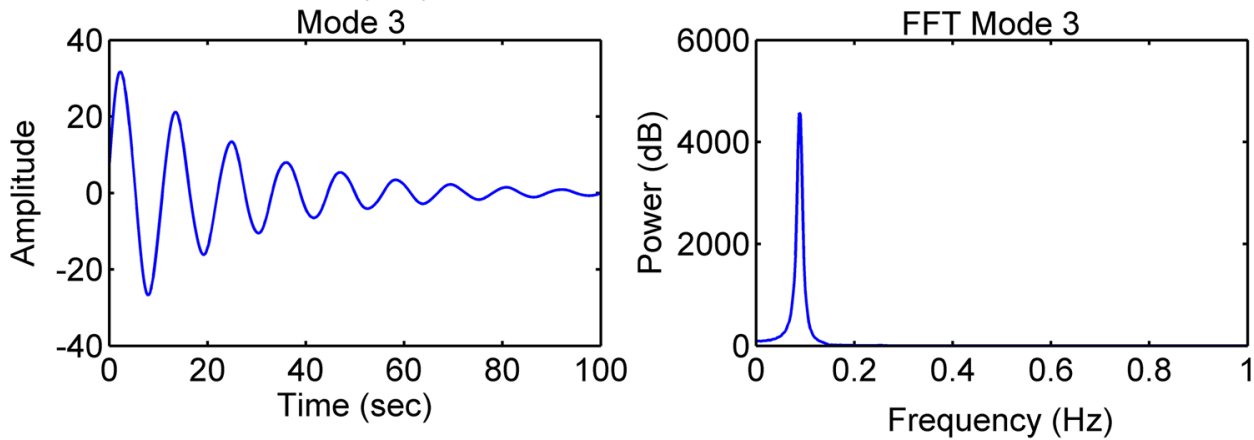

$\mathbf{M}=\left[\begin{array}{lll}1 & 0 & 0 \\ 0 & 2 & 0 \\ 0 & 0 & 1\end{array}\right]$

$\mathbf{K}=\left[\begin{array}{ccc}5 & -1 & 0 \\ -1 & 4 & -3 \\ 0 & -3 & 3.5\end{array}\right]$

$\mathbf{C}=\alpha \mathbf{M}=\alpha\left[\begin{array}{lll}1 & 0 & 0 \\ 0 & 2 & 0 \\ 0 & 0 & 1\end{array}\right]$

$M=\left[\begin{array}{lll}3 & 0 & 0 \\ 0 & 2 & 0 \\ 0 & 0 & 1\end{array}\right]$

$\mathrm{K}=\left[\begin{array}{ccc}4 & -2 & 0 \\ -2 & 4 & -2 \\ 0 & -2 & 10\end{array}\right]$

The initial conditions used in free excitation are changed to $x(0)=\left[\begin{array}{lll}0 & 0 & 0\end{array}\right]^{T}$ and $\dot{x}(0)=\left[\begin{array}{lll}0 & 0 & 1\end{array}\right]^{T}$, the remaining parameters were left the same as used in proportional damping case. Fairly accurate modal identification results are obtained in closely spaced modes shown in Tables 4 and 5, respectively.

A highly damped system ( $\alpha=0.13$ ) with closely spaced mode in free excitation is shown in Fig. 5. The closely spaced 2 nd and 3rd modes of the system responses that can be hardly judged in the power spectral densities are clearly decoupled by the CP model as shown in Fig. 6.

\subsection{Non-proportional damping}

The parameters of a system under non-proportional damping are given as follows,
$C=\left[\begin{array}{ccc}0.3856 & 0.2290 & -0: 9702 \\ 0.2290 & 0.5080 & -0: 0297 \\ -0.9702 & -0.0297 & 0.3241\end{array}\right]$

The model parameters are obtained by slightly changing the damping matrix used in Ref. [21] that results in complex modes. McNeil and Zimmerman [21] presented a standard method to transform the complex modes into real ones due to the output of the $\mathrm{CP}$ model to provide a real value demixing matrix. Tables 6 and 7 show the identification results. Fairly well comparison can be seen among the identified and the theoretical results. Equation (23) can be used to evaluate the accuracy of the identified mode shapes. The system responses and recovered modal responses are shown in Figs. 7 and 8 , respectively. Little influence on the output of the $C P$ algorithm has been seen in case of non-proportional 
Fig. 4 Recovered modes for 3DOF system in stationary random excitation (proportional damping)

Table $3 \mathrm{CP}$ identified values in $10 \%$ root-mean-square noise $(\alpha=0.08)$

Table 4 CP Identified results for free excitation (closely spaced modes)
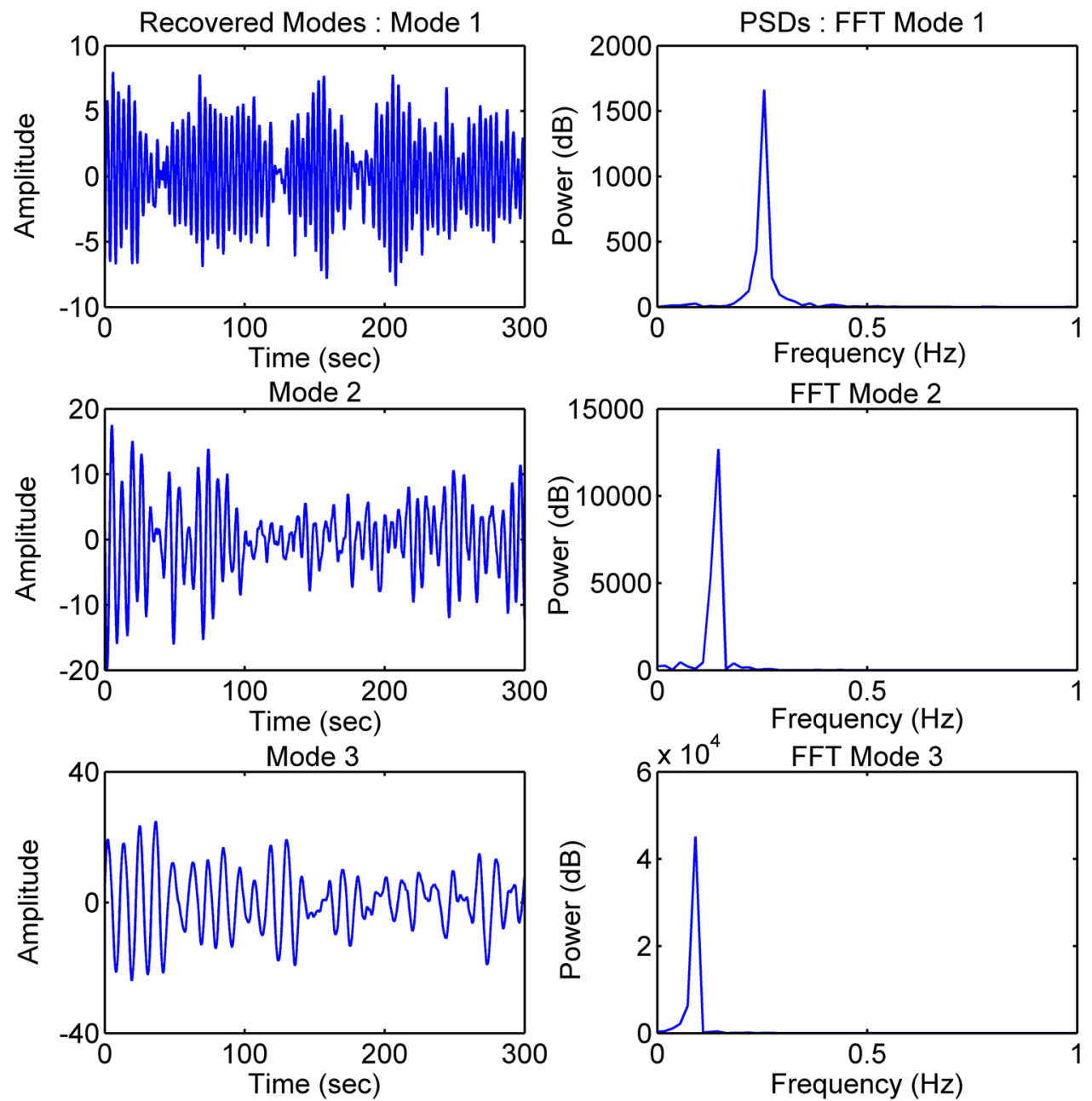

\begin{tabular}{lllllll}
\hline Mode & \multicolumn{2}{l}{ Frequency $(\mathrm{Hz})$} & & \multicolumn{2}{l}{ Damping ratio (\%) } & \multirow{2}{*}{ MAC values } \\
\cline { 2 - 3 } & Theoretical value & CP identified & & Theoretical value & CP identified & \\
\hline 1 & 0.0894 & 0.0885 & & 4.4437 & 4.2151 & 0.9989 \\
2 & 0.1457 & 0.1459 & & 2.7299 & 2.7910 & 0.9617 \\
3 & 0.2521 & 0.2529 & & 1.5775 & 1.5343 & 0.9979 \\
\hline
\end{tabular}

\begin{tabular}{|c|c|c|c|c|c|c|c|}
\hline \multirow[t]{2}{*}{ Mode } & \multirow[t]{2}{*}{ Comparison } & \multicolumn{3}{|c|}{ Frequency $(\mathrm{Hz})$} & \multicolumn{3}{|c|}{ Damping ratio (\%) } \\
\hline & & 1 & 2 & 3 & 1 & 2 & 3 \\
\hline \multirow[t]{2}{*}{$a=0.08$} & Theoretical & 0.1039 & 0.3425 & 0.3713 & 3.8279 & 1.1618 & 1.0715 \\
\hline & CP identified & 0.1074 & 0.3418 & 0.3711 & 3.8199 & 1.1434 & 1.0151 \\
\hline \multirow[t]{2}{*}{$a=0.13$} & Theoretical & 0.1039 & 0.3425 & 0.3713 & 9.9526 & 3.0208 & 2.7860 \\
\hline & CP identified & 0.1074 & 0.3418 & 0.3711 & 9.9770 & 2.9906 & 2.7274 \\
\hline
\end{tabular}


Table 5 Modal assurance criterion results in closely space mode cases

\begin{tabular}{|c|c|c|c|c|c|c|c|c|c|}
\hline \multirow[t]{2}{*}{ Mode } & \multicolumn{3}{|c|}{ Free excitation } & \multicolumn{3}{|c|}{$\begin{array}{l}\text { Stationary Gaussian white } \\
\text { noise }\end{array}$} & \multicolumn{3}{|c|}{$\begin{array}{l}\text { Non-stationary Gaussian } \\
\text { white noise }\end{array}$} \\
\hline & 1 & 2 & 3 & 1 & 2 & 3 & 1 & 2 & 3 \\
\hline$a=0.08$ & 1.000 & 0.997 & 0.999 & 0.999 & 1.000 & 0.999 & 1.000 & 0.997 & 0.996 \\
\hline$a=0.13$ & 1.000 & 0.973 & 0.975 & 0.999 & 0.999 & 1.000 & 0.999 & 0.976 & 0.991 \\
\hline
\end{tabular}

Fig. 5 System responses for 3DOF system in free excitation (closely spaced modes)
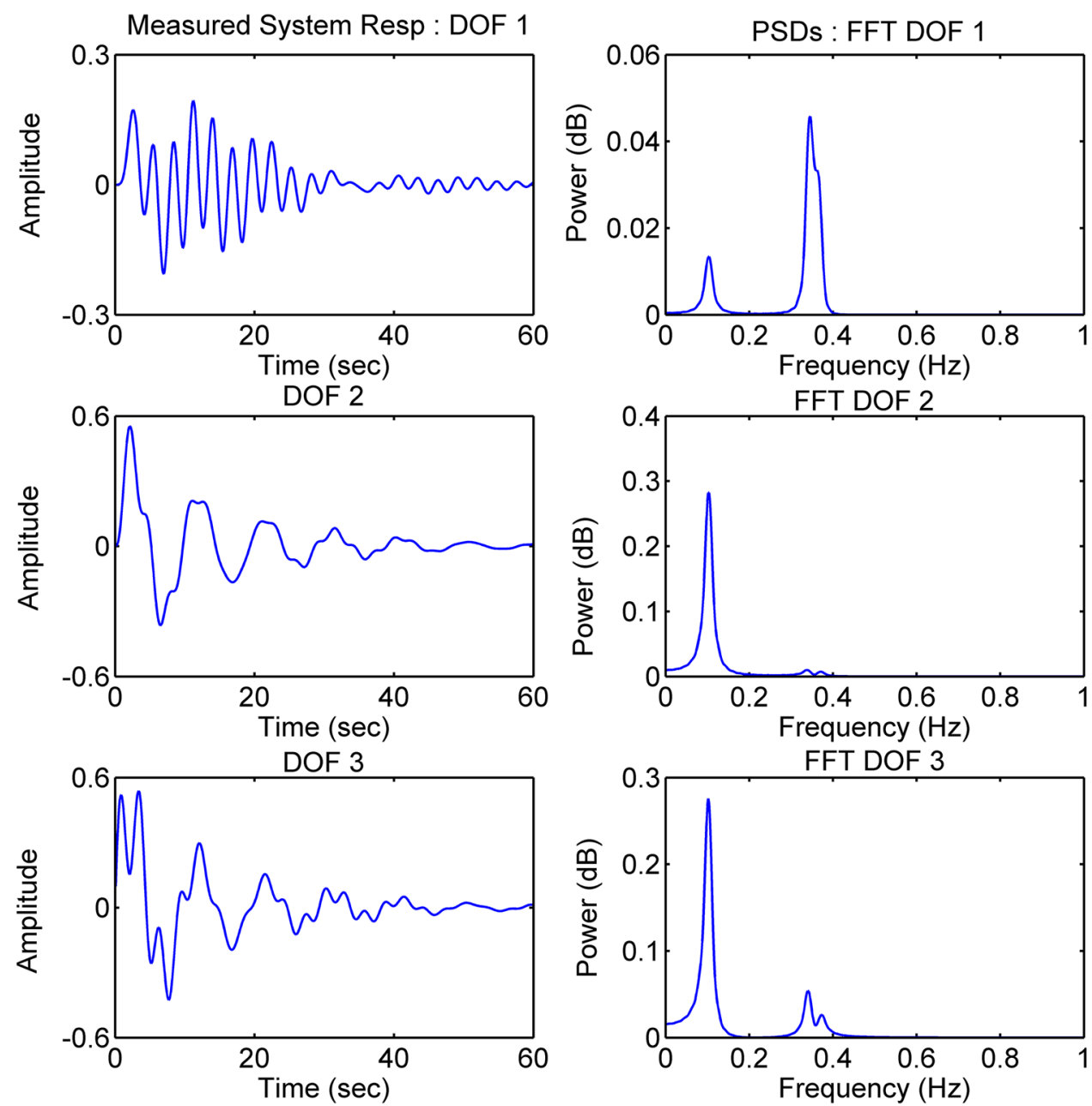

damping; still it offers better approximation for complex modes.

\subsection{Modal identification of a 12-DOF system}

The performance of complexity pursuit algorithms is further extended towards large scale structures, a 12-DOF system is built up such that the values of constant mass matrix $\mathbf{M}$ are given by; $\mathrm{m}_{1}=2, \mathrm{~m}_{2} \ldots, \mathrm{m}_{11}=1, \mathrm{~m}_{12}=3$, and values of stiffness matrix $\mathbf{K}$ are $k_{1}, k_{2}, \ldots k_{13}=20000$ and damping matrix is calculated by $\mathbf{C}=\alpha \mathbf{M}$ with $\alpha=3$, where $\alpha$ is the damping ratio. The first mode has a theoretical damping ratio of $4.46 \%$. The frequencies of the 12 modes are distributed between 5.3505 and $44.5827 \mathrm{~Hz}$; with a sampling frequency set to $1000 \mathrm{~Hz}$. The system is excited at the 12th DOF, and the time histories of the signals with 5000 samples are measured (the length of the signal can be increased and the accuracy holds). Modal assurance criterion (MAC) values for all 12 modes under different conditions are shown in Table 8. A high correlation among the approximated modes can be seen with (MAC) values above 0.99 for all damping levels. 
Fig. 6 Modal responses for 3DOF system in free excitation (closely spaced modes)
Table 6 Identified results of free excitation in nonproportional high damping

Table 7 Identified MAC values for non-proportional high damping
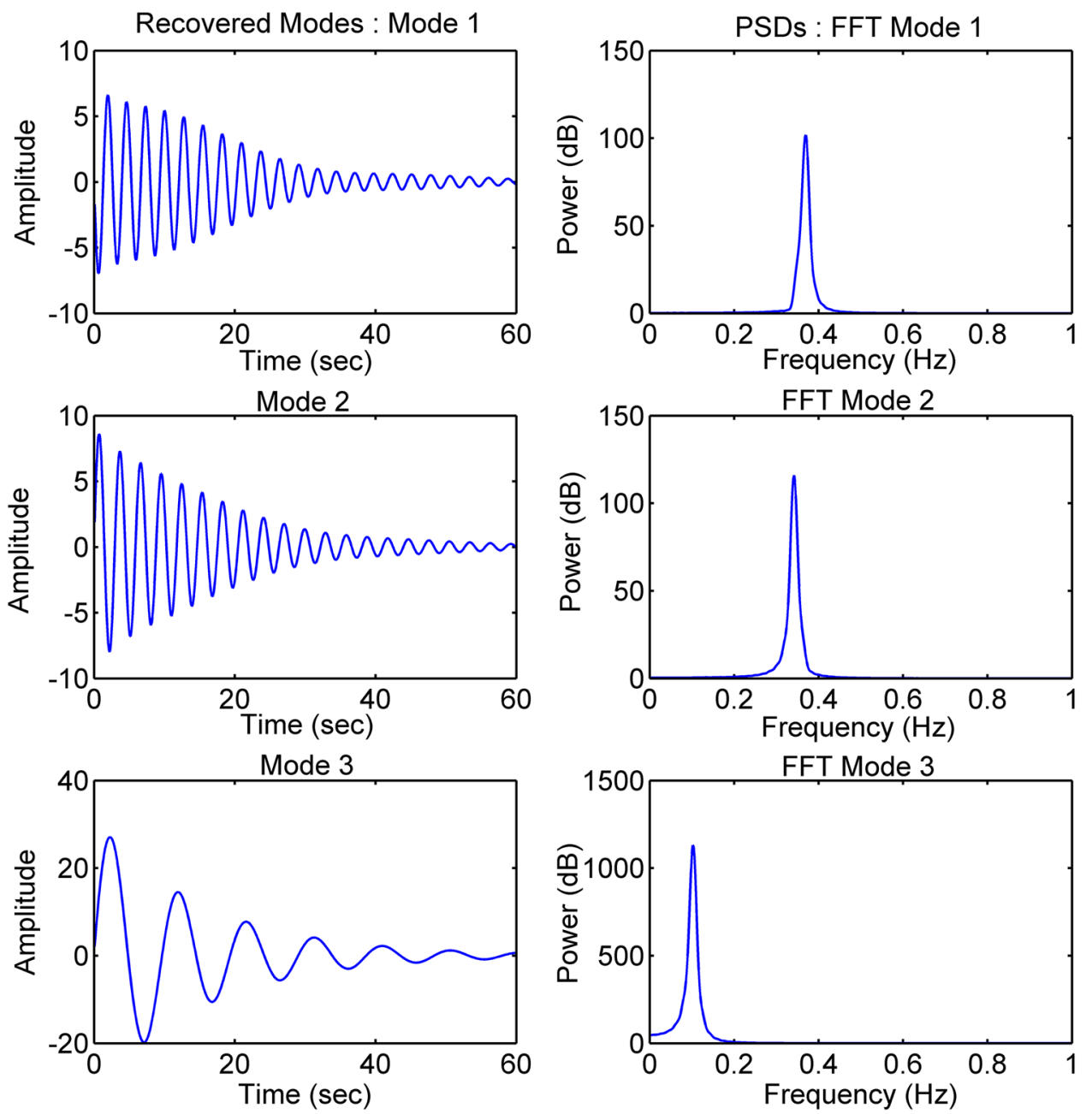

\begin{tabular}{|c|c|c|c|c|c|}
\hline \multirow[t]{2}{*}{ Mode } & \multicolumn{2}{|c|}{ Frequency $(\mathrm{Hz})$} & \multicolumn{2}{|c|}{ Damping Ratio (\%) } & \multirow{2}{*}{$\begin{array}{l}\text { Modal } \\
\text { assurance } \\
\text { criterion }\end{array}$} \\
\hline & CP identified & Theoretical & CP identified & Theoretical & \\
\hline 1 & 0.137 & 0.135 & 10.95 & 10.88 & 0.985 \\
\hline 2 & 0.239 & 0.244 & 6.731 & 6.875 & 0.952 \\
\hline 3 & 0.497 & 0.508 & 4.674 & 4.872 & 0.981 \\
\hline
\end{tabular}

\begin{tabular}{|c|c|c|c|c|c|c|c|c|c|}
\hline \multirow[t]{2}{*}{ Mode } & \multicolumn{3}{|c|}{ Free excitation } & \multicolumn{3}{|c|}{$\begin{array}{l}\text { Stationary Gaussian white } \\
\text { noise }\end{array}$} & \multicolumn{3}{|c|}{$\begin{array}{l}\text { Non-Stationary Gaussian } \\
\text { white noise }\end{array}$} \\
\hline & 1 & 2 & 3 & 1 & 2 & 3 & 1 & 2 & 3 \\
\hline$a=0.13$ & 1.000 & 0.973 & 0.975 & 0.999 & 0.999 & 1.000 & 0.999 & 0.976 & 0.991 \\
\hline
\end{tabular}


Fig. 7 System responses for 3DOF system in free excitation (non-proportional damping)
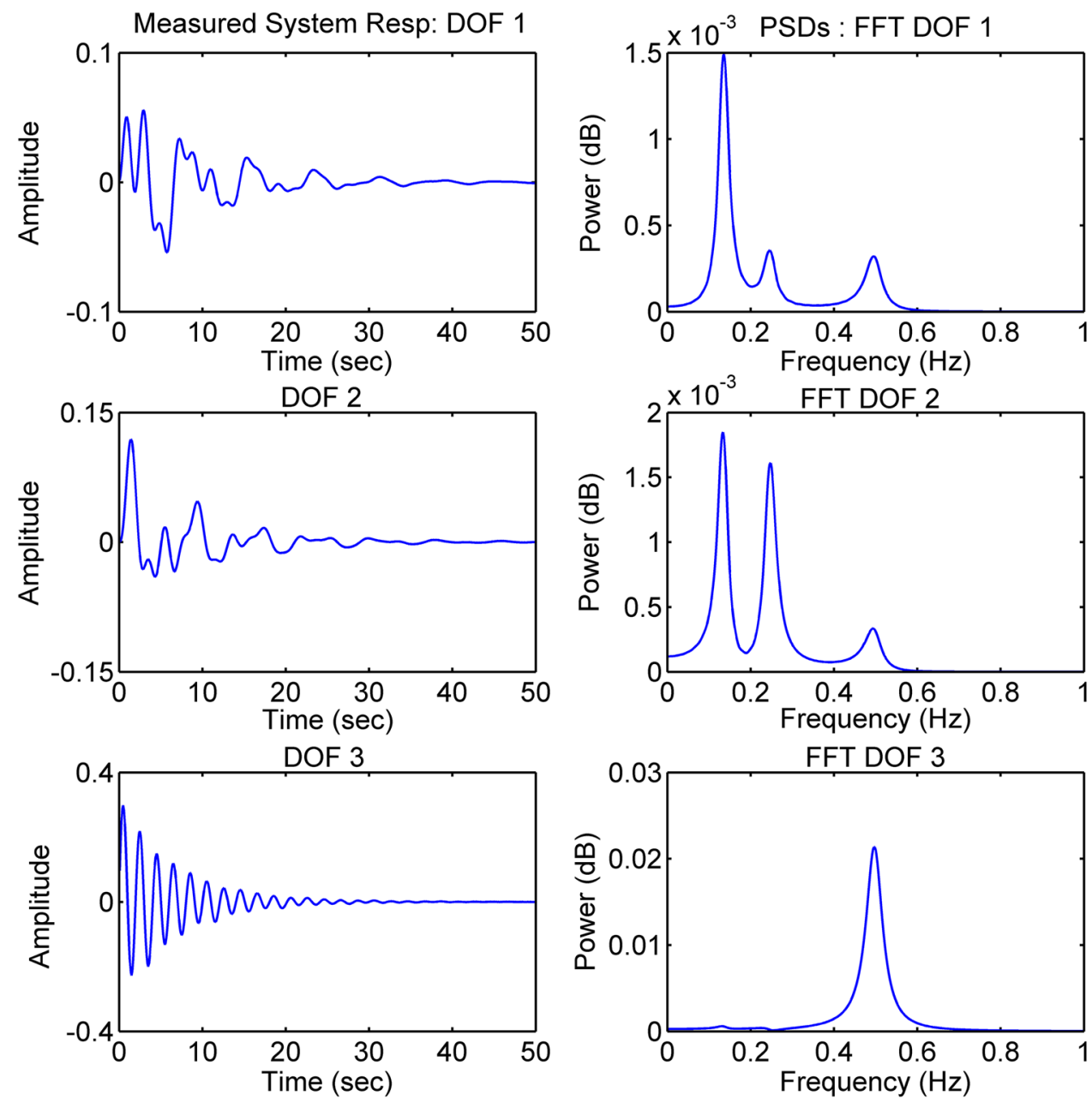

\section{Experimental analysis}

\subsection{Measurement probe to detect magnetic field of a pipeline}

Figure 9a shows the block diagram of the data measurement system. It contains a pair of batteries $(12 \mathrm{~V}$ and $24 \mathrm{~V}$ ) connected as source to all power equipments, a magnetic probe, National Instruments data acquisition unit, industrial control computers, and a global positioning system. The position of the underground pipeline is located at each point of the measured distance with the help of a global positioning system (GPS). Figure $9 \mathrm{~b}$ illustrates the schematic image of the magnetic probe having five triaxial magnetic field sensors, with a sensor to sensor baseline distance of $0.1 \mathrm{~m}$. The analog data collected by each sensor are comprised of $x, y$ and $z$-components of the magnetic field. The measured analog signals are converted into digital form by the National data acquisition system, which is further transferred to an industrial control computer. This part of measurement system is a computer (CPU) and a storage unit that records the real time data to be used for further processing.

\subsection{Indoor experimental procedure}

The indoor experimental data is recorded for a pipeline test sample made of Q235 steel with a measurement length of $10 \mathrm{~m}$, inner wall to wall diameter of $0.1 \mathrm{~m}$ and wall thickness of $0.002 \mathrm{~m}$. Figure 10a shows a two-story robot carrying the magnetic probe across the length of the pipeline with probe to pipeline distance of $1 \mathrm{~m}$. The National Instruments data acquisition unit has been fixed at the second story above the magnetic probe. The defects on the wall of the pipeline can be seen in Fig. 10b, i.e. a groove of depth $0.001 \mathrm{~m}$ and a hole of diameter $0.01 \mathrm{~m}$ at a distance of $4 \mathrm{~m}$ from each other. The sampling frequency is set to $1 \mathrm{kHz}$. The measurement system data are arranged in a $m \times n$ matrix, with $m=15$ signals and $n=120,000$ samples.

The CP-BSS method is applied on the measured data matrix. The recovered modal responses for $x, y$ and $z$ components of magnetic field for sensor 1 and senor 3 are 
Fig. 8 Modal responses for 3DOF system in free excitation (non-proportional damping)
Table 8 MAC values for 12-DOF system proportional damping
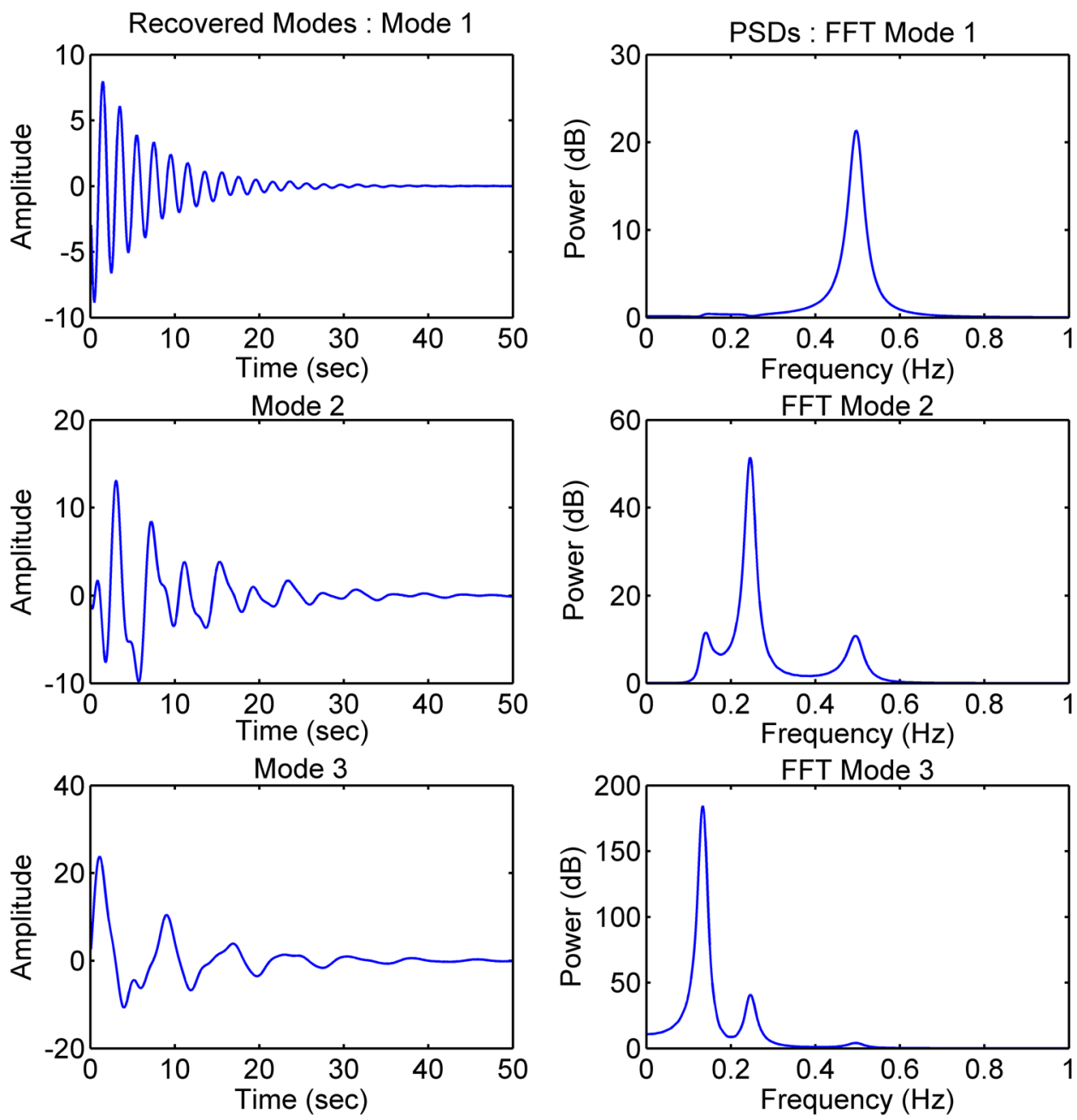

\begin{tabular}{|c|c|c|c|c|c|c|c|c|c|}
\hline \multirow[t]{2}{*}{ Modes } & \multicolumn{3}{|c|}{ Free excitation } & \multicolumn{3}{|c|}{ Stationary GWN } & \multicolumn{3}{|c|}{ Non-stationary GWN } \\
\hline & $a=1$ & $a=2$ & $a=3$ & $a=1$ & $a=2$ & $a=3$ & $a=1$ & $a=2$ & $a=3$ \\
\hline 1 & 9950 & 0.9972 & 0.9904 & 0.9989 & 0.9946 & 0.9837 & 0.9973 & 0.9975 & 0.9938 \\
\hline 2 & 9974 & 0.9977 & 0.9939 & 0.9989 & 0.9951 & 0.9857 & 0.9970 & 0.9979 & 1.0000 \\
\hline 3 & 0.9994 & 0.9952 & 0.9922 & 0.9982 & 0.9981 & 0.9937 & 0.9989 & 0.9948 & 0.9921 \\
\hline 4 & 0.9997 & 0.9977 & 0.9959 & 0.9995 & 0.9982 & 0.9927 & 0.9996 & 0.9974 & 0.9958 \\
\hline 5 & 0.9999 & 0.9992 & 0.9985 & 0.9999 & 0.9995 & 0.9991 & 0.9998 & 0.9991 & 0.9985 \\
\hline 6 & 0.9998 & 0.9997 & 0.9995 & 0.9999 & 0.9996 & 0.9996 & 0.9998 & 0.9997 & 0.9995 \\
\hline 7 & 0.9999 & 0.9998 & 0.9997 & 1.0000 & 0.9998 & 0.9997 & 0.9999 & 0.9998 & 0.9997 \\
\hline 8 & 1.0000 & 0.9999 & 0.9999 & 1.0000 & 1.0000 & 0.9999 & 1.0000 & 0.9999 & 0.9999 \\
\hline 9 & 1.0000 & 1.0000 & 1.0000 & 1.0000 & 1.0000 & 1.0000 & 1.0000 & 1.0000 & 1.0000 \\
\hline 10 & 1.0000 & 1.0000 & 0.9999 & 1.0000 & 1.0000 & 0.9999 & 1.0000 & 1.0000 & 0.9999 \\
\hline 11 & 1.0000 & 1.0000 & 0.9999 & 1.0000 & 1.0000 & 0.9999 & 1.0000 & 1.0000 & 0.9999 \\
\hline 12 & 1.0000 & 1.0000 & 0.9999 & 1.0000 & 1.0000 & 0.9999 & 1.0000 & 1.0000 & 0.9999 \\
\hline
\end{tabular}

given in Figs. 11 and 12, respectively. The results clearly indicate that the measured system data are accurately transformed into their respective modal responses. The power spectral densities calculated from the recovered mode matrix shows the abrupt variation in signal power at the given frequencies of $25 \mathrm{~Hz}$ and $75 \mathrm{~Hz}$ in all three powers spectral densities which is due to the damage 
a

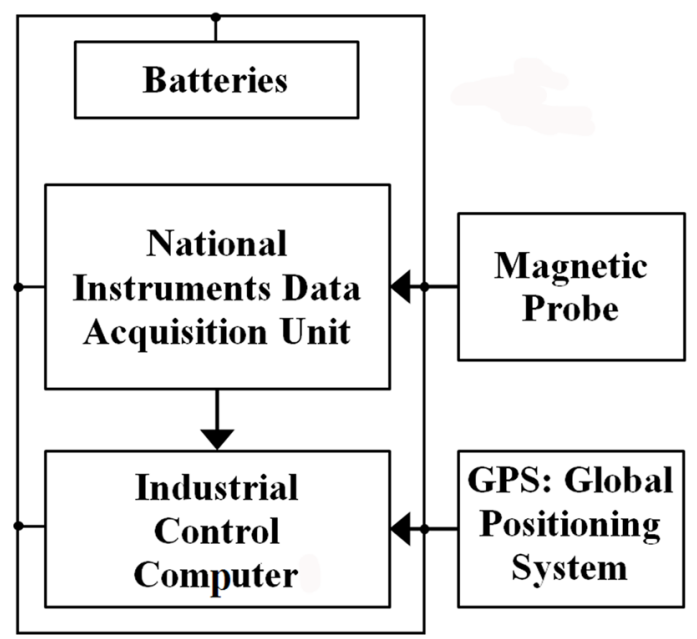

b

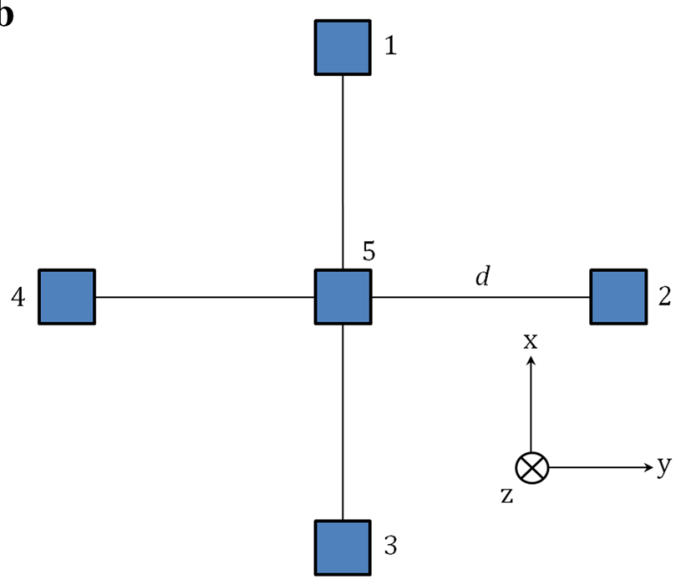

Fig. 9 a Block diagram of the measurement system $\mathbf{b}$ magnetic probe sensor array

occurring in the pipeline i.e. groove and hole present in the pipeline test sample.

\subsection{Outdoor experimental setup}

An outdoor experiment has been performed on an underground pipeline in Hebei province of China. The pipeline is made from Q235 steel with a diameter of $0.323 \mathrm{~m}$ and a wall thickness of $0.005 \mathrm{~m}$. The detection height is about $1.5 \mathrm{~m}$ and the sampling frequency is $100 \mathrm{~Hz}$. Figure 13 shows the pipeline data detection route.

The recovered modal responses for sensor 1 along with their respective power spectral densities are shown in Fig. 14. The identified visible modes are shown as the active modes present in the pipeline structural response data. The power spectral densities calculated from the recovered modes clearly reveals the frequency and
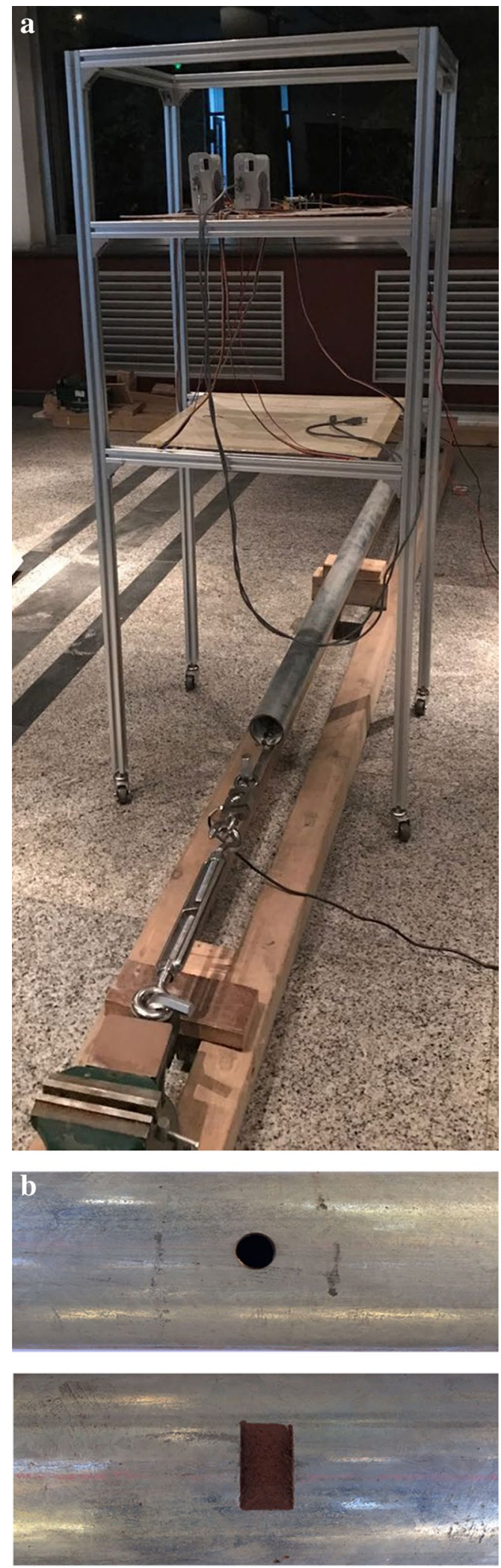

Fig. 10 a Magnetic probe passing through pipeline b Pipeline groove and hole defects 
Fig. 11 Recovered modal responses for $x, y$ and $z$-components of magnetic field detected at sensor 1 and their respective power spectral densities

Fig. 12 Recovered modal responses for $x, y$ and z-components of magnetic field detected at sensor 3 and their respective power spectral densities
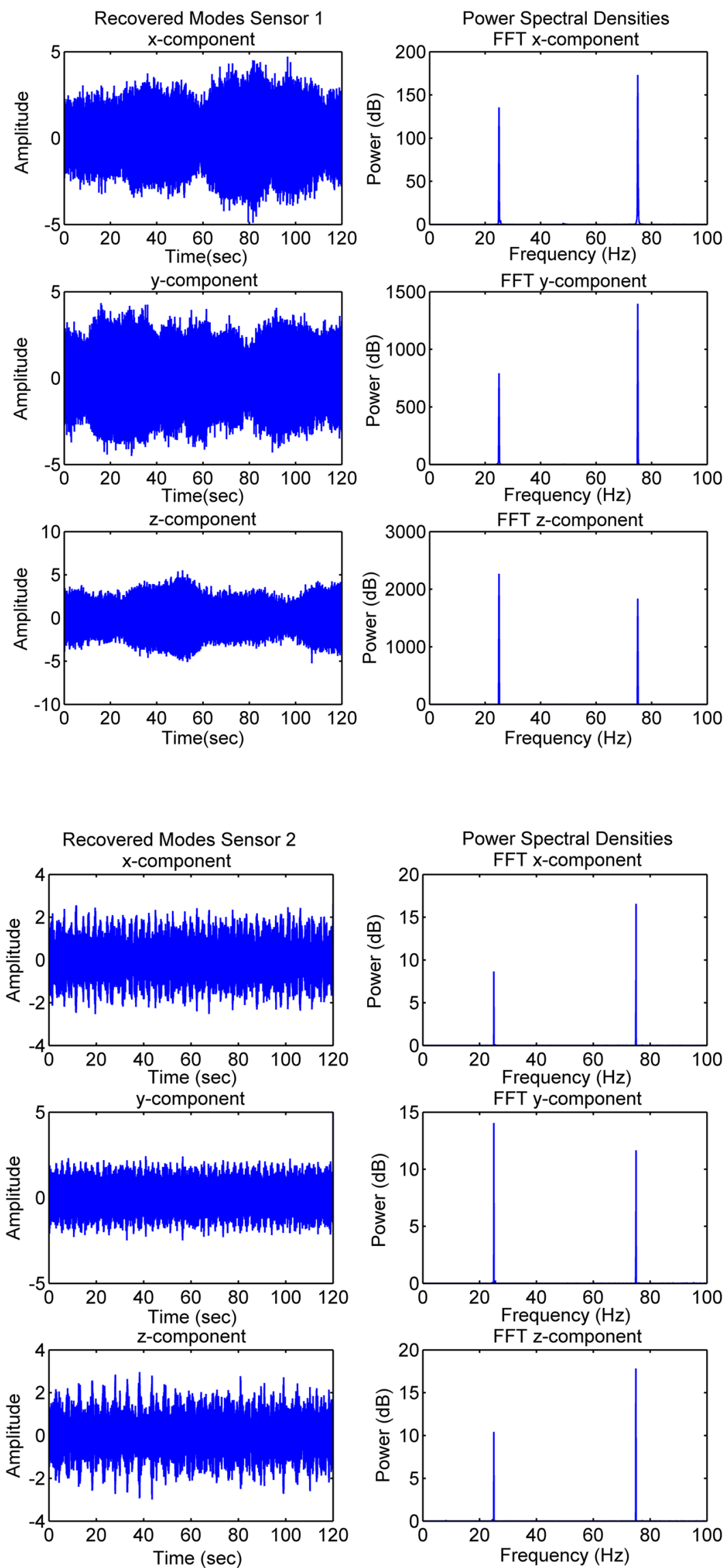


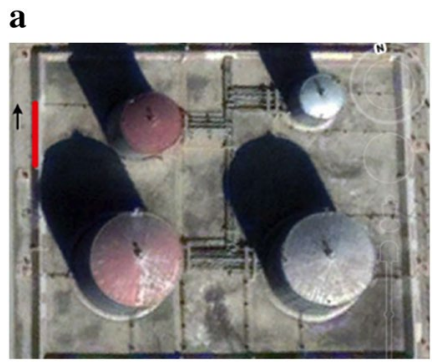

b

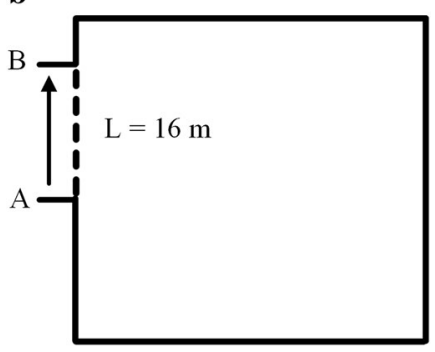

Fig. 13 a Image of the Rinqiu area in Hebei province of China, the red line shows the data collection route $\mathbf{b}$ schematic image of the pipeline data detection route with a measured length of $16 \mathrm{~m}$ starting from point $A$ to point $B$

power of the source signals with abrupt variation due to damage occurring in the pipeline. The results demonstrate that the active modes present in the pipeline magnetic field can be accurately identified using the complexity pursuit based blind identification model.

\section{Concluding remarks}

Time based damage detection of underground ferromagnetic pipelines is presented in this paper. Complexity Pursuit based blind signal separation algorithms are implemented to identify structural damage from the measured magnetic field sensor data. Using Fast Fourier transform the power spectral densities are calculated from the approximated modal responses. Numerical simulations for multi-DOF systems are carried out to elaborate the CP based BSS method under different damping conditions. The complexity pursuit based BSS model is implemented on the indoor and outdoor experimental data comprised of 3-axis magnetic field signals; it offers excellent results about the pipeline structural information. The proposed method requires minimum user interaction because the parameters of the model remain same throughout the process of targeting the input data. Similarly length of the recorded sensor data does not influence the accuracy of the CP model. The performance of the unsupervised CPBSS model to identify structural information makes it more
Fig. 14 Recovered modal responses for magnetic field ( $x$, $y$ and z-components) recorded at sensor 1 and their power spectral densities
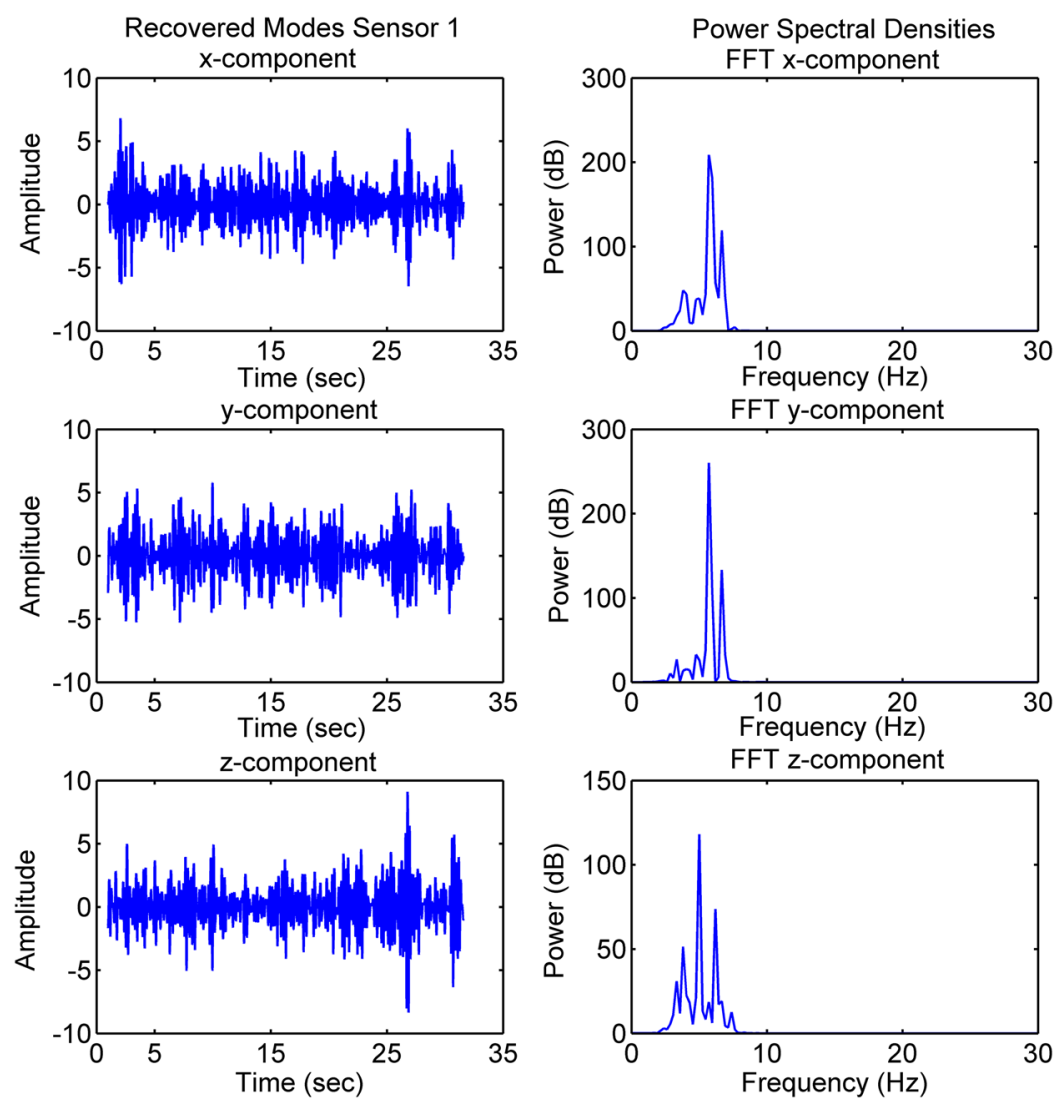

SN Applied Sciences A SPRINGER NATURE journal 
suitable for real time as well as for off-line inspection of underground ferromagnetic pipeline structures.

Funding The work is supported by The National Key Research and Development Program of China (2017YFC0805005), Joint Program of Beijing Municipal Natural Science Foundation Commission and Beijing Municipal Education Commission (18JH0005), China Postdoctoral Science Foundation (2018T110018), Collaborative Innovation Project of Beijing Chaoyang District (CYXC1709) and "Rixin Scientist" of the Beijing University of Technology.

\section{Compliance with ethical standards}

Conflict of interest The authors declare that they have no conflict of interest.

\section{References}

1. Teixeira AP, Soares CG, Netto TA, Estefen SF (2008) Reliability of pipelines with corrosion defects. Int J Press Vessels Pip 85:228-237

2. Cunha SB, Pasqualino IP, Pinheiro BC (2009) Stress-life fatigue assessment of pipelines with plain dents. Fatigue Fract Eng Mater Struct 32:961-974

3. Dubov AA (1997) A study of metal properties using the method of magnetic memory. Met Sci Heat Treat 39:401-405

4. Wang ZD, Yao K, Deng B, Ding KQ (2010) Theoretical studies of metal magnetic memory technique on magnetic flux leakage signals. NDT and E International 43:354-359

5. Li C, Chen C, Liao K (2015) A quantitative study of signal characteristics of non-contact pipeline magnetic testing. Insight Non Destr Test Cond Monit 57:324-330

6. Sharma R, Vignolo L, Schlotthauer $G$, Colominas MA, Rufiner $\mathrm{HL}$, Prasanna SRM (2017) Empirical mode decomposition for adaptive AM-FM analysis of speech: a review. Speech Commun 88:39-64

7. Yang JN, Lei Y, Lin S, Huang N (2004) Hilbert-Huang based approach for structural damage detection. ASCE J Eng Mech 130:85-95

8. Huang N, Shen Z, Long S, Wu M, Shih H, Zheng Q, Yen N, Tung C, Liu H (1998) The empirical mode decomposition and the Hilbert spectrum for nonlinear and non-stationary time series analysis. Proc R Soc Lond Ser A Math Phys Eng Sci 454:903-995

9. Kijewski T, Kareem A (2003) Wavelet transforms for system identification in civil engineering. Comput Civ Infrastruct Eng 18:339-355

10. Hazra B, Narasimhan S (2010) Wavelet-based blind identification of the UCLA Factor building using ambient and earthquake responses. Smart Mater Struct 19:025005

11. Daubechies I (1992) Ten lectures on wavelets. In: CBMS-NSF conference series in applied mathematics, SIAM

12. Reza R, Farnam G, Milad H, Mohammad P (2018) Quantifying prestressing force loss due to corrosion from dynamic structural response. J Sound Vib 433:129-137

13. Lu ZR, Law SS (2006) Identification of prestress force from measured structural responses. Mech Syst Signal Process 20(8):2186-2199
14. Law SS, Lu ZR (2005) Time domain responses of a prestressed beam and prestress identification. J Sound Vib 288(4-5):1011-1025

15. Poncelet F, Kerschen G, Golinval JC, Verhelst D (2007) Outputonly modal analysis using blind source separation techniques. Mech Syst Signal Process 21(6):2335-2358

16. Castiglione R, Antoni J, Garibaldi L (2018) Separation and identification of structural modes in largely underdetermined scenarios using frequency banding. J Sound Vib 414:192-217

17. Hyvärinen A, Oja E (2000) Independent component analysis: algorithms and applications. Neural Netw 13:411-430

18. Belouchrani A, Abed-Meraim AK, Cardoso JF, Moulines E (1997) A blind source separation technique using second-order statistics. IEEE Trans Signal Process 45:434-444

19. Antoni J, Chauhan S (2013) A study and extension of secondorder blind source separation to operational modal analysis. $J$ Sound Vib 332(4):1079-1106

20. Chauhan S, Martell R, Allemang RJ, Brown DL (2007) Application of independent component analysis and blind source separation techniques to operational modal analysis. In: Proceedings of the 25th IMAC, Orlando (FL), USA

21. McNeill SI, Zimmerman DC (2008) A framework for blind identification using joint approximate diagonalization. Mech Syst Signal Process 22(7):1526-1548

22. Kerschen G, Poncelet F, Golinval JC (2007) Physical interpretation of independent component analysis in structural dynamics. Mech Syst Signal Process 21:1561-1575

23. Ullah Z, Xinhua W, Yingchun C, Tao Z, Haiyang J (2018) Blind identification of pipeline damage using independent component analysis with wavelet transform. In: CSAA/IET international conference on aircraft utility systems, p 7. https://doi. org/10.1049/cp.2018.0314

24. Stone JV (2001) Blind source separation using temporal predictability. Neural Comput 13:1559-1574

25. Hyvärinen A (2001) Complexity pursuit: separating interesting components from time series. Neural Comput 13:883-898

26. Yang Y, Nagarajaiah S (2013) Blind modal identification of output-only structures in time-domain based on complexity pursuit. Earthq Eng Struct Dyn 42(13):1885-1905

27. Antoni J, Castiglione R, Garibaldi L (2017) Interpretation and generalization of complexity pursuit for the blind separation of modal contributions. Mech Syst Signal Process 85:773-788

28. Doebling SW, Farrar CR, Prime M., Shevitz DW (1996) Damage identification and health monitoring of structural and mechanical systems from changes in their vibration characteristics: a literature review. Rep. LA-13070-MS, UC-900, Los Alamos National Laboratory, Los Alamos, NM

29. Yang Y, Nagarajaiah S (2014) Structural damage identification via a combination of blind feature extraction and sparse representation classification. Mech Syst Signal Process 45:1-23

30. Yang Y, Nagarajaiah S (2015) Output-only modal identification by compressed sensing: non-uniform low-rate random sampling. Mech Syst Signal Process 56-57:15-34

31. Xie S, He Z, Fu Y (2005) A note on stone's conjecture of blind source separation. Neural Comput 17:321-330

Publisher's Note Springer Nature remains neutral with regard to jurisdictional claims in published maps and institutional affiliations. 